\title{
The MACHO PROJECT: MICROLENSING AND VARIABLE STARS
}

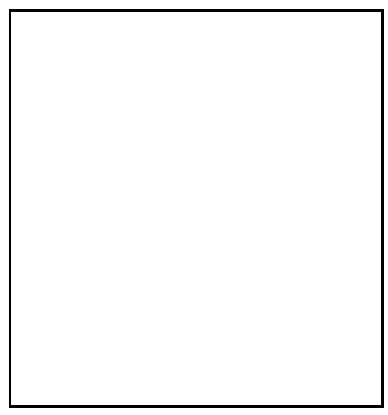

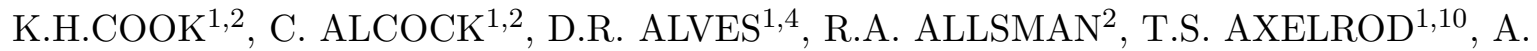

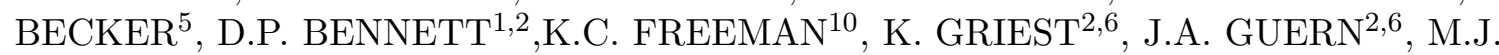
LEHNER $^{2,6}$, S.L. MARSHALL ${ }^{1,2}$, D. MINNITI ${ }^{1}$, B.A. PETERSON ${ }^{10}$, M.R. PRATT ${ }^{1,5}$, P.J. QUINN $^{7}$, A.W. RODGERS ${ }^{10}$, C.W. STUBBS ${ }^{5}$, W. SUTHERLAND ${ }^{8}$, D.L. WELCH ${ }^{9}$

(The MACHO Collaboration)

${ }^{1}$ Lawrence Livermore National Laboratory, Livermore, CA 94550

${ }^{2}$ Center for Particle Astrophysics, University of California, Berkeley, CA 94720

3 Supercomputing Facility, Australian National Univ., Canberra, ACT 0200, Australia

${ }^{4}$ Department of Physics, University of California, Davis, CA 95616

${ }^{5}$ Department of Physics and Astronomy, University of Washington, Seattle, WA 98195

${ }^{6}$ Department of Physics, University of California San Diego, La Jolla, CA 92093-0350

7 European Southern Observatory, D-85748 Garching bei München, Germany

8 Department of Physics, University of Oxford, Oxford OX1 3RH, U.K.

${ }^{9}$ Dept. of Physics and Astronomy, McMaster Univ., Hamilton, Ontario, Canada L8S $4 M 1$

10 Mount Stromlo and Siding Springs Obs., Australian Natl. Univ., Weston, ACT 2611, Australia

\begin{abstract}
The MACHO Project monitors millions of stars in the Large Magellanic Cloud, the Small Magellanic Cloud and the bulge of the Milky Way searching for the gravitational microlensing signature of baryonic dark matter. This Project has yielded surprising results. An analysis of two years of data monitoring the Large Magellanic Cloud points to $\sim 50 \%$ of the mass of the Milky Way's halo in compact objects of $\sim 0.5 M_{\odot}$. An analysis of one year of monitoring the bulge has yielded more microlensing than predicted without the invocation of a massive bar or significant disk dark matter. The huge database of light curves created by this search is yielding information on extremely rare types of astrophysical variability as well as providing temporal detail for the study of well known variable astrophysical phenomena. The variable star catalog created from this database is previewed and example light curves are presented.
\end{abstract}

\section{Introduction}

The MACHO Project is monitoring millions of stars every night searching for the gravitational microlensing signature of massive compact halo objects (Machos). This project has the dedicated use 
the Great Melbourne Telescope at Mount Stromlo. This endeavor was stimulated by Paczyński's suggestion 18] that gravitational microlensing was a possible way to detect baryonic dark matter in the halo of the Milky Way. The principle of microlensing is simple; if a Macho lies near the line of sight to a background star (the source), it will deflect light from the source and produce two images. For galactic scales, these images cannot be resolved even by HST $(\sim 0.001$ arcsec and thus microlensing), but the two unresolved images combine to give an apparent increase in the source brightness. Due to the relative motions of the observer, lens and source, this magnification is transient, so the effect appears as a symmetrical and unique brightening in an otherwise constant star. The duration of the event is a function of the mass of the lens, the relative distances of the lens and source and the motion of the lens with respect to the line of sight. The magnification is just a function of the distance of the lens from the line of sight. The probability that a source will be microlensed is termed the optical depth to microlensing, $\tau$.

The Large Magellanic Cloud (LMC) was chosen as the primary target because its line of sight passes through much of the halo yet it is relatively nearby, and contains millions of stars resolvable in modest seeing with a small telescope. Our secondary target was chosen as the Galactic bulge for two reasons. Although the LMC is circumpolar from Mount Stromlo, it is at too high an airmass to be usefully observed when the bulge is overhead. Calculations before we began taking data [13] suggested that there should be an observable microlensing signal from known stellar populations in the disk and the bulge so observing the bulge would serve to prove whether microlensing could be detected with our system. We also observe the Small Magellanic Cloud (SMC) at a lower priority. The SMC provides a different line of sight through the halo, but is sufficiently farther away and smaller than the LMC that we can only monitor a few million stars with our system.

\section{Instrumentation, Observations, and Reductions}

The refurbishment of the 1.27-m Great Melbourne Telescope has been described by Hart et al. [14. Figure 1 shows in detail the corrector, beam splitter and CCD cameras which allow us to monitor 0.5 square deg simultaneously in two passbands. These two passbands were chosen to maximally utilize the quantum efficiency of our thick CCDs while cutting out much of the sky brightness due to $\mathrm{OH}$ emission in the red and scattering in the blue. Figure 1 also shows the total throughput as a function of wavelength for our two cameras. Each camera contains a 2x2 mosaic of 2048x2048 CCDs. Exposure times are $300 \mathrm{~s}$ toward the LMC, $150 \mathrm{~s}$ toward the bulge and $600 \mathrm{~s}$ toward the SMC. It takes about 70 s to read out the 32 Mpixels. Details of the camera system can be found in [27].

Images are flattened immediately upon acquisition, then simultaneously archived to 8-mm tape and photometry begun. Reductions and analysis are done in the dome using two Sparc 1000s and a large disk farm (currently $\sim 200$ Gbyte). MACHO field centers are defined so that the 0.5 square degree fields tile the denser regions of the LMC, SMC and much of the southeast quadrant of the bulge within 10 degrees of the Galactic center. These field centers (as well as much additional information on the MACHO Project) can be found at URL http://wwwmacho.anu.edu.au. Our observing strategy is to sequentially image each of these fields, spiraling outward from dense bar fields in the LMC and generally moving from west to east in the bulge. As of September 1996, over 48,000 exposures have been taken with the system, of which about $60 \%$ are of the LMC. The data covered in this presentation is derived from the central 22 fields in the LMC observed for the first two years of the project (September 1992 to November 1994) and a central 24 fields in the bulge observed in the project's first bulge season (1993).

Deep images from the early months of the project have been reduced to create photometry templates. A template is divided into 64 'chunks' in each color and relatively bright, unblended stars in each chunk are chosen as fiducials to define the point spread function (PSF) and astrometric reference frame in future observations. A routine observation is reduced chunk by chunk by determining the geometric transformation from the template to the current chunk using the fiducial stars, defining a PSF using the fiducials, and fitting at each transformed template position (more details can be found 


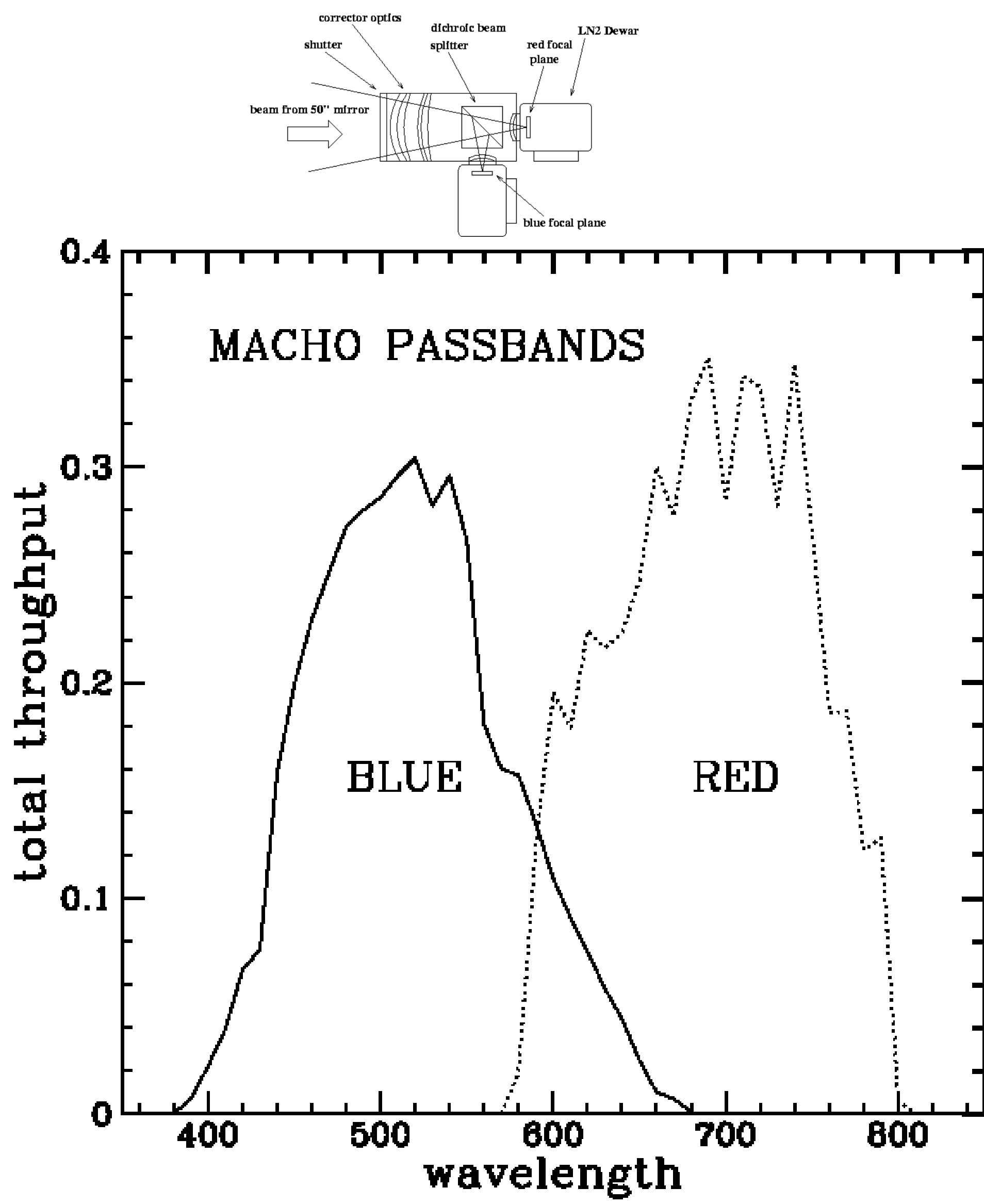

Figure 1: The left panel shows the telescope optics which re-image the $f / 4.5$ primary beam to $f / 3.9$ and produce a 1 degree corrected field. The right panel shows the total throughput as a function of wavelength for the red and blue cameras of the MACHO Project. 
in [3] and [6]). This scheme allows rapid reductions, and a whole night of observations can be reduced within a few hours of dawn. Although the microlensing search is effected using only relative photometry, it is useful to derive transformations to standard astronomical passbands. This is complicated by two systematic effects: 1) the template observation for each field is subject to different extinction, seeing, and possible cloud obscuration, and 2) the photometric zero point varies from chunk to chunk (see [6]) within a template observation due to systematic effects of the photometry code. (This is due to the fact that there is no effort made to derive an aperture correction for individual chunk reductions.) We have obtained roughly 30 photometric nights of CCD data using the CTIO 0.9-m and SSO 1-m to measure the necessary 1408 transformations (64 chunks per field and 22 fields). Although the analysis of this data is not complete, we have determined a mean transformation to Johnson $\mathrm{V}$ and Kron-Cousins $\mathrm{R}$ which we will use in this paper. This transformation yields magnitudes good to about $\pm 0.15 \mathrm{mag}$ and colors which are good to about $\pm 0.1 \mathrm{mag}$ (the zero point offsets in the two passbands are correlated). Light curves of time-ordered photometry are then built for all template objects. Light curves are not created for objects which are not in the template. These light curves are then analyzed for evidence of microlensing [3] [4] [6]. Because photometry is available within hours of an observation, a star's current magnitude can be compared to its mean magnitude determined from a previous analysis and if a previously constant star demonstrates a significant deviation, our software produces an alert. These alerts have been used to notify observers around the world that microlensing events are in progress (see http://darkstar.astro.washington.edu).

Table 1. Optical Depth Estimates

\begin{tabular}{|l|l|l|}
\hline Data Set & $\tau\left(10^{-7}\right)$ & $95 \%$ CL \\
\hline LMC: 8 events & 2.93 & $1.47-5.28$ \\
LMC: 6 events & 2.06 & $0.93-4.00$ \\
LMC: no short events & $<0.4$ & $0.00-0.4$ \\
Bulge: all stars & 24.3 & $16.9-33.3$ \\
Bulge: clump giants & 39.2 & $29.0-70.9$ \\
\hline
\end{tabular}

\section{Microlensing Results}

We have detected significant microlensing toward both the LMC and the bulge. Our microlensing discoveries are important for three reasons: 1) we have detected significant microlensing by halo dark matter looking toward the LMC, 2) we have detected no short 1 microlensing events looking toward the LMC and so have ruled out a significant fraction of the halo being composed of objects with mass $2.5 \times 10^{-6}-8.0 \times 10^{-2} M_{\odot}$, and 3 ) we have detected a higher optical depth for microlensing toward the bulge than predictions based on simple Milky Way models, lending support to the presence of a significant bar.

It is important to note that the microlensing interpretation for the events we report has been strongly supported and can be considered proven. The majority of events are well fit by the simple functional form needed to describe point source, point lens microlensing. There have been spectroscopic observations through the course of microlensing toward the bulge [10] and the LMC [7] which have shown no spectral changes during apparent brightness changes of more than one magnitude in the span of a week. The distribution of magnifications has been shown to be that expected from the uniform distribution of impact parameters which result from microlensing [ [- Ex]. Exceptions to simple lensing support the microlensing interpretation because they are predicted deviations due to the motion of the earth [2], due to the finite size of stars [24], and due to the existence of binary lenses [30] [9]. While there are deviations from simple lensing due to the blending of lensed and unlensed images, this is well understood and can be accounted for by fitting an unmagnified fraction of the baseline flux, and thus adding an additional fit parameter in each color [3] [6]. 


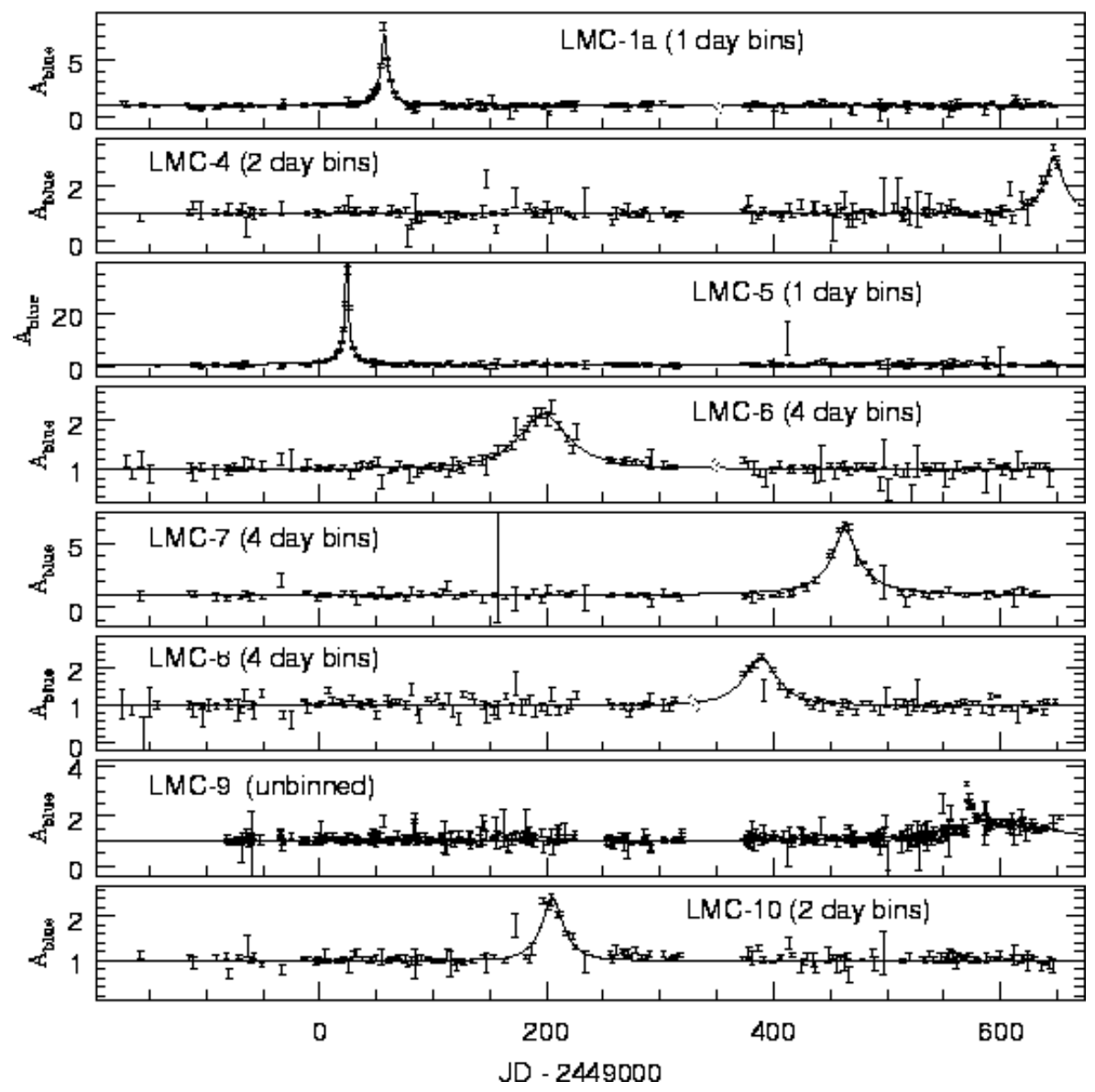

Figure 2: The 8 LMC microlensing events are plotted as time versus relative blue camera flux normalized to the mean unlensed flux $\left(\mathrm{A}_{\text {blue }}\right)$. The solid line is the fit to simple microlensing and the data has been averaged in bins appropriate to the event duration. LMC-9 is due to lensing by a binary as described in [9].

We have recently concluded an analysis of the first two years of data on 22 fields in the LMC. These data have yielded nine microlensing candidates eight of which are shown in Figure 2. The position of the baseline color and magnitude for these 8 events is shown in a composite color-magnitude diagram of LMC data in Figure 3. The ninth event occurred in an unresolved source star which was lensed during the template observation. Because our efficiency analysis does account for sources which are not represented by objects in our template, this event is not considered further. The measured optical depths for various samples in both the LMC and the bulge are shown in Table 1.

A fraction more than one microlensing event would be expected from known populations in the Milky Way and the LMC after monitoring $8.6 \times 10^{6}$ stars for two years. Thus, we considered two samples for analysis, a six event sample (excluding one possible LMC-LMC event and one additional event) and an eight event sample. Both samples produce similar conclusions. We detect about half the predicted optical depth if the dark halo were composed of only Machos. A maximum likelihood analysis suggests that we are detecting $\sim 50 \%$ of the dark halo mass in objects of $\sim 0.5 \mathrm{M}_{\odot}$. A full account of this analysis is forthcoming [6]. This analysis and a companion analysis looking for evidence of short microlensing events in our data [5] support and extend the EROS negative result [8] demonstrating the absence of short microlensing events. Our result limits objects in the mass range $2.5 \times 10^{-6}-8.0 \times 10^{-2} M_{\odot}$ (Venus through brown dwarf masses) to contributing less than $20 \%$ of the dark halo.

Although the MACHO Project has only analyzed one season of bulge data to date, this season 


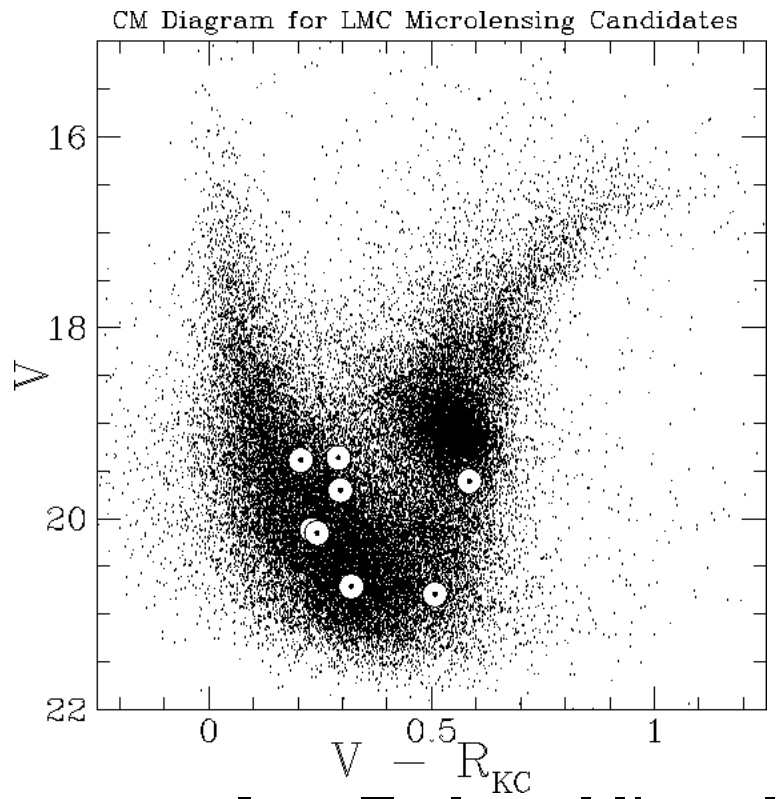

CM Diagram for Bulge Microlensing Candidates

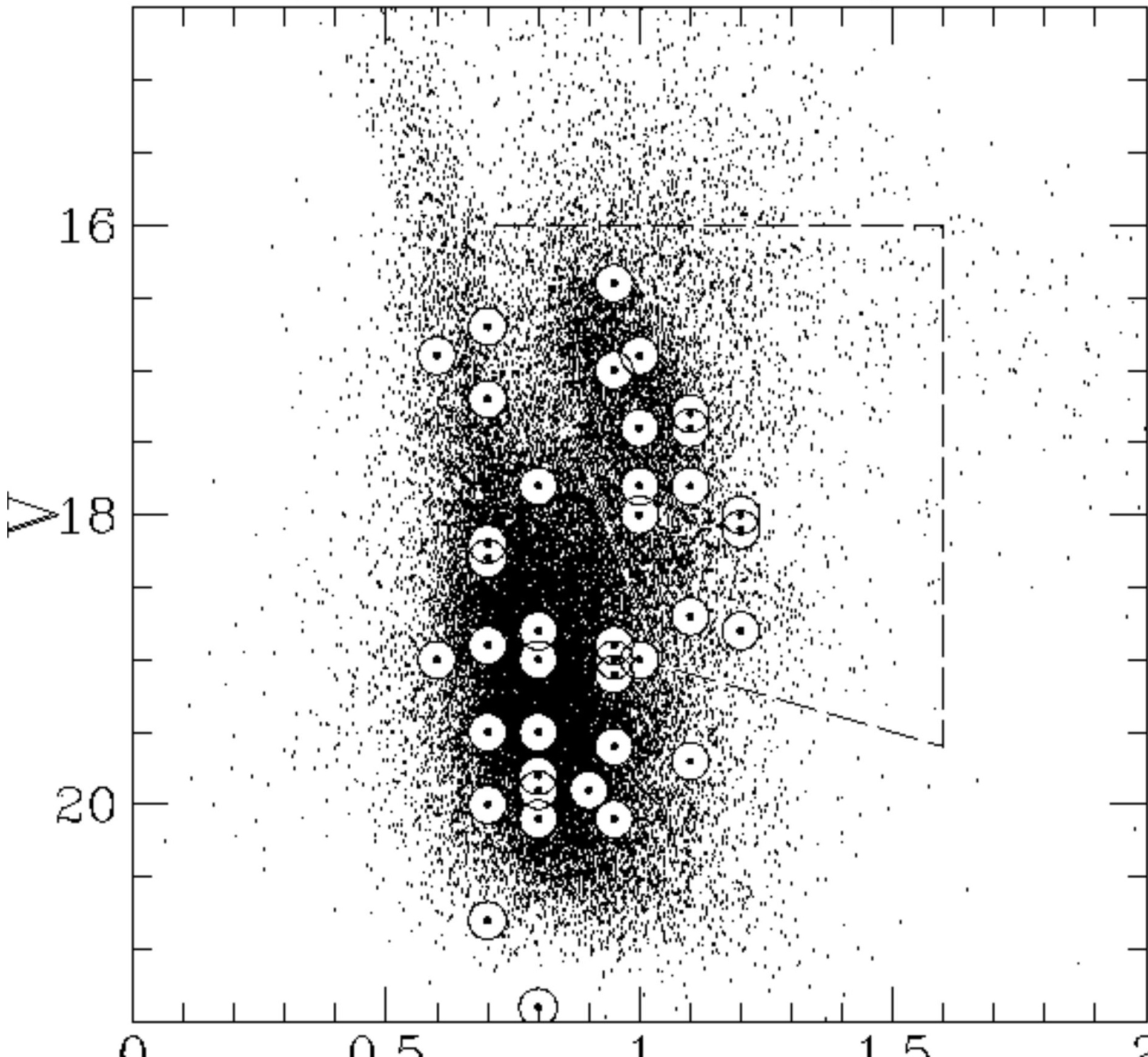


yielded 42 microlensing candidates. We have detected an additional 75 bulge events with our real time alert system since late 1994. We have used both the full 1993 sample and a subset derived from bright, clump giant source stars to calculate an optical depth to the bulge. Because detailed efficiency simulations have not been completed for our bulge data, the corrections for faint source stars are somewhat uncertain, but the clump giant sample is not subject to significant efficiency corrections. Both samples yield a higher optical depth than the expected $1 \times 10^{-6}$ optical depth to the bulge 13. [19]. OGLE and DUO report similar measurements of optical depth [29] [1]. These data support the hypothesis of a massive bar in the Milky Way and may also provide evidence of significant disk dark matter [20] [4]. Microlensing of apparent disk stars as evidenced by the microlensing events along the upper blue sequence in Figure 3 underscores the possibility of significant disk dark matter.

\section{Variables in the MACHO Database}

The color-magnitude diagrams (CMDs) of Figure 3 demonstrate the photometry product of the microlensing search. The mean photometric error at $\mathrm{V}=20$ for hundreds of epochs is about $25 \%$ for the LMC and about $40 \%$ for the bulge. The LMC CMD is created from less than $1 \%$ of the LMC light curves while the bulge CMD is created from about $1 \%$ of the bulge light curves. Much of the scatter in these diagrams is due to the uncertainty in the transformations as discussed earlier. As part of the analysis of the light curves in the search for microlensing, a variability statistic is calculated for each light curve. The $\chi^{2} /$ dof for a fit to a constant is calculated using only the $80 \%$ of points closest to the median for each light curve. The light curve is defined as variable if this, robust, $\chi^{2} /$ dof is greater than the regular $\chi^{2} /$ dof which can occur by chance $1 \%$ of the time for a constant light curve. The nightly sampling for extended periods of time results in an effective sampling even for variables with periods much shorter than a day. This is because the sampling interval constantly changes since we attempt to observe each field at its lowest airmass in a night.

Of the $\sim 9$ million LMC light curves from the first two years of LMC data 37,051 light curves were identified as variable. Of the $\sim 12$ million bulge light curves currently analyzed from 1993, 38,718 were identified as variable. An additional analysis of LMC light curves was conducted to identify those which were rejected because of relatively rare excursions below the robust envelope defined above. This analysis identified about 1000 more eclipsing binaries, 3000 more low amplitude RR Lyrae (primarily due to confusion), and about 6000 low amplitude, low signal-to-noise variable candidates. These 85,000 light curves were tested for periodic behavior using a code designed specifically for the MACHO Project [25]. When the red and blue light curve yielded the same period to within $0.1 \%$ the light curve was defined as representing a periodic variable. Figure 4 shows period-amplitude and period-magnitude plots for the 23,589 periodic variables identified in the LMC. Figure 5 shows the period-amplitude and period-magnitude plots for the 14,776 periodic variables identified in the bulge. A preliminary description of the LMC variables has been presented [11].

These figures hold a wealth of information about the variable stellar populations of the LMC and the bulge. There are also certain systematic features in this data set which are apparent. There are vertical lines in the distributions for two reasons due to the phasing code we have used. The phasing code will return an alias of an integer fraction of a day when it tries to phase a low signal-to-noise (low amplitude) aperiodic variable. For high signal-to-noise aperiodic or very long period variables, it will fold them with a period of the light curve length. There are multiple, long period vertical lines because different fields yielded different light curve durations. We have used these figures to define regions in period, magnitude, amplitude space which contain highly purified samples of particular types of variables. We have also used the blue-to-red amplitude ratio to discriminate pulsating from eclipsing variables [17]. One can clearly see the RRab and RRc in the LMC as distinct clumps near $\log ($ period $)=-0.3$ and -0.5 , respectively. In the bulge, the RRc locus is clearly contaminated by short period, low amplitude variables, mostly eclipsing binaries. The fundamental and overtone Cepheid period-luminosity (PL) loci stand out in the LMC data and are absent in the bulge data as would be expected because of its mostly old population. Perhaps the most striking feature of these diagrams 
is the appearance of clear, multiple PL relations for bright, long period variables. These are all giants lying just beyond the tip of the giant branch. As can be seen, they have low amplitudes with a suggestion of increasing amplitude with increasing period. Eclipsing binaries are found scattered across these diagrams. More detailed discussions of the $\sim 10,000$ RR Lyrae [17], 1500 Cepheids in the LMC [31], and Type II Cepheids and RV Tauri in the LMC [22] contained in the MACHO database are presented elsewhere in this volume.

Examples of some of the more interesting periodic variables from our database are shown in Figure 6. Eclipsing binaries from the EROS and OGLE survey are discussed in detail elsewhere in this volume [26] [28] [21]. Examples of extremes in period for eclipsing systems in the MACHO data are shown in the left panels. About a dozen eclipsing, red giant pairs with periods over 100 days have been identified in our LMC data so far. Two examples of the low amplitude, periodic red giant variables with periods less than 100 days are shown in the middle panels. There seems to be quite a range in the constancy of the amplitude of stars in this region of the period-amplitude diagram. The relatively short periods and the sinusoidal light curves are suggestive of overtone oscillations. Although there are few classical Cepheids along our line of sight to the bulge, we have discovered about 20 new BL Her and W Vir stars. Examples of two of these are shown in right panels.

We have also begun a detailed examination of the aperiodic blue variables found in the LMC data. Our interest in these stars was stimulated by the observation of rare, small amplitude outbursts in mostly constant stars which we have termed bumpers. We have compiled a catalog of about 400 aperiodic blue variables which demonstrate some truly odd photometric behavior. These stars were chosen from about 250,000 light curves with $14.0<V<18.0$ and $(V-R)<0.3$ [23]. Characteristic light curves of two major classes of variability found in the catalog have been previously presented [11]. Figure 7 shows three other interesting blue, aperiodic variables: an odd one, a background Seyfert and a nova. In this set of aperiodic blue variables we have also found supersoft X-ray sources, and planetary nebulae.

\section{Summary}

The unevenly spaced sampling, the wide field of view, the simultaneous color information and the long, densely sampled light curves make the MACHO database a treasure trove for the study of time variability of astrophysical sources. While we have only begun to examine this database outside the context of the search for Machos, the early returns promise much for the future. The MACHO Project is also moving rapidly toward achieving its goal of determining the baryonic content of the halo. The MACHO Project plans to continue operation at least through 1999. At the end of this time, we expect to have a sample of about 50 microlensing events across the face of the LMC, about 1000 events toward the bulge, and a database of light curves for more than 50 million stars in the LMC, the SMC and the bulge, spanning 8 years.

Acknowledgements. We are very grateful for the skilled support by the technical staff at MSO. Work at LLNL is supported by DOE contract W7405-ENG-48. Work at the CfPA is supported NSF AST-8809616 and AST-9120005. Work at MSSSO is supported by the Australian Department of Industry, Technology and Regional Development. WJS is supported by a PPARC Advanced Fellowship. KG thanks support from DOE OJI, Sloan, and Cottrell awards. CWS thanks support from the Sloan, Packard and Seaver Foundations.

\section{References}

[1] Alard, C. 1995, ESO Messenger, 80, 31

[2] Alcock, C., et al. (The MACHO Collaboration) 1995, ApJ, 454, L125 
[3] Alcock, C., et al. (The MACHO Collaboration) 1996, ApJ, 461, 84

[4] Alcock, C., et al. (The MACHO Collaboration) 1996, ApJ, in press and astro-ph/9512146

[5] Alcock, C., et al. (The MACHO Collaboration) 1996, ApJ, in press and astro-ph/9604176

[6] Alcock, C., et al. (The MACHO Collaboration) 1996, ApJ, in press and astro-ph/9605148

[7] Alcock, C., et al. (The MACHO Collaboration) 1996, in preparation

[8] Auborg, E., et al. (The EROS Collaboration), 1995, AA 301, 1

[9] Bennett, D. et al. (The MACHO Collaboration) 1996, astro-ph/960612

[10] Bennetti, S., et al., 1995 AA, 294, L37

[11] Cook, K. H. et al. (The MACHO Collaboration) 1995, ASP Conf. Proc., 83, 221

[12] Cowley, A., et al, 1984, AJ, 286, 196

[13] Griest, K., et al (The MACHO Collaboration) 1991, ApJ, 366, 412

[14] Hart, J. et al (The MACHO Collaboration), 1996, PASP, 108, 220

[15] Liller, W., 1992, IAUC 5651

[16] Marshall, S. Ph.D. Thesis, UC Santa Barabara

[17] Minniti, D., et al (The MACHO Collaboration) these proceedings

[18] Paczyński, B. 1986, ApJ, 304, 1

[19] Paczyński, B. 1991, ApJ, 371, L63

[20] Paczyński, B., 1994, ApJ, 435, L113

[21] Paczyński, B. 1996, these proceedings

[22] Pollard, K. A., et al (The MACHO Collaboration), these proceedings

[23] Purdue, P., et al (The MACHO Collaboration), 1995, BAAS 27, 1402

[24] Pratt, M., et al. (The MACHO Collaboration) 1995, BAAS, 27, 1351

[25] Reimann, J. Ph.D. Thesis, UC Berkeley

[26] Rucinski 1996, these proceedings

[27] Stubbs, C., et al (The MACHO Collaboration), 1993, SPIE Proc. 1900, 192

[28] Tobin, W., 1996, these proceedings

[29] Udalski, A., et al. 1994, Acta Astron., 44, 165

[30] Udalski, A., et al. 1994, ApJ, 435, L113

[31] Welch, D., et al (The MACHO Collaboration), these proceedings 


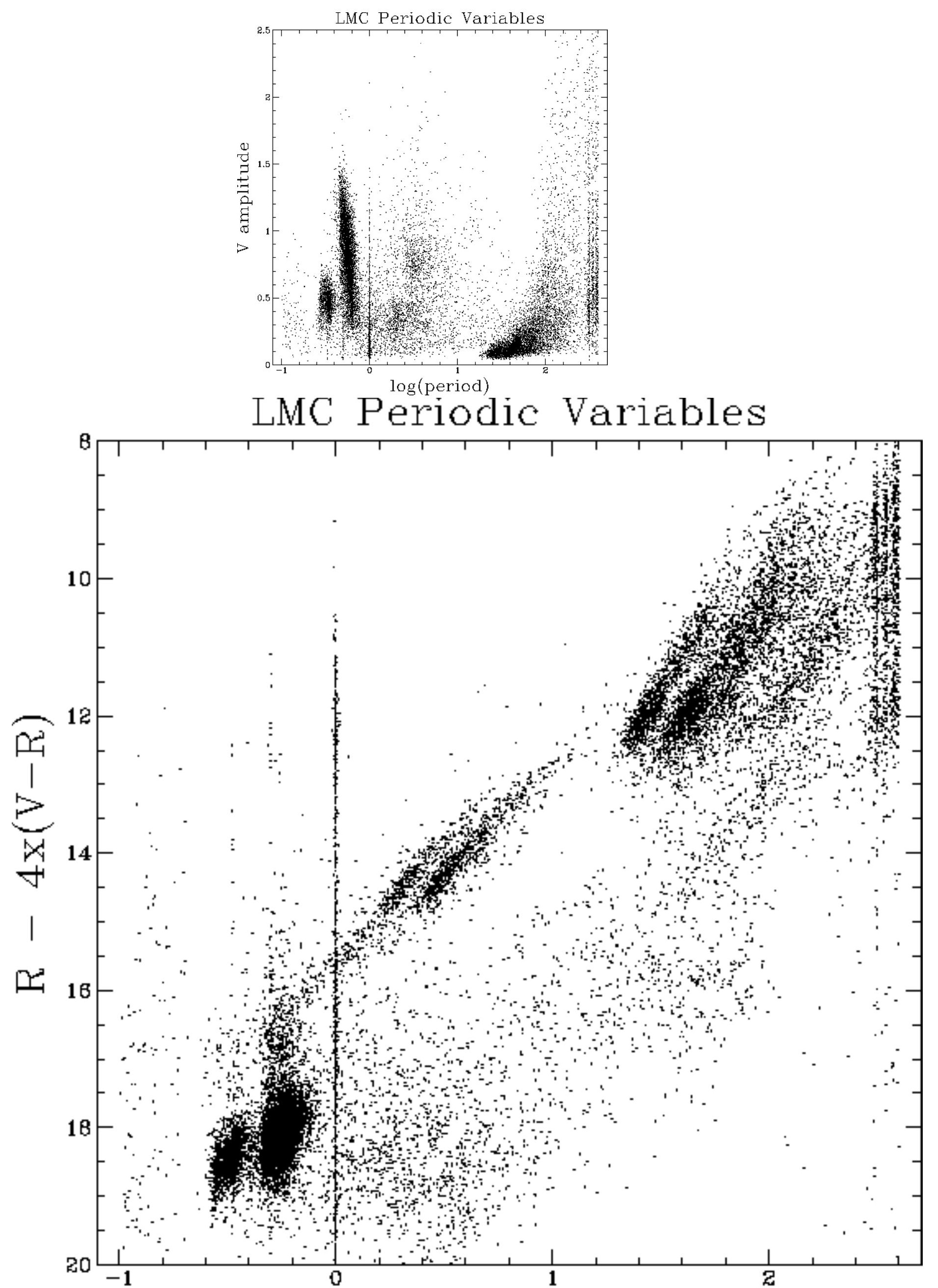




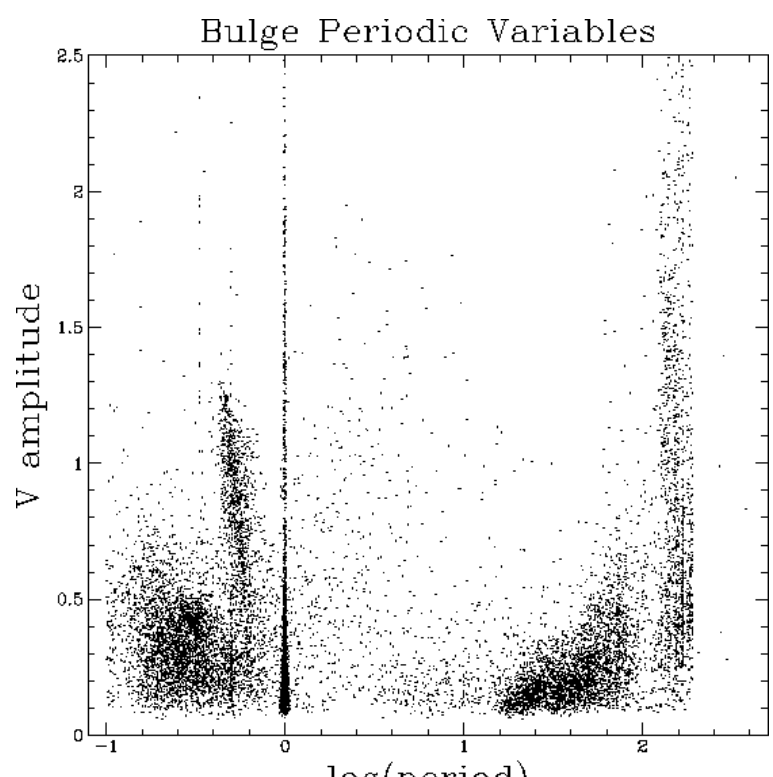

Bulge Periodic Variables

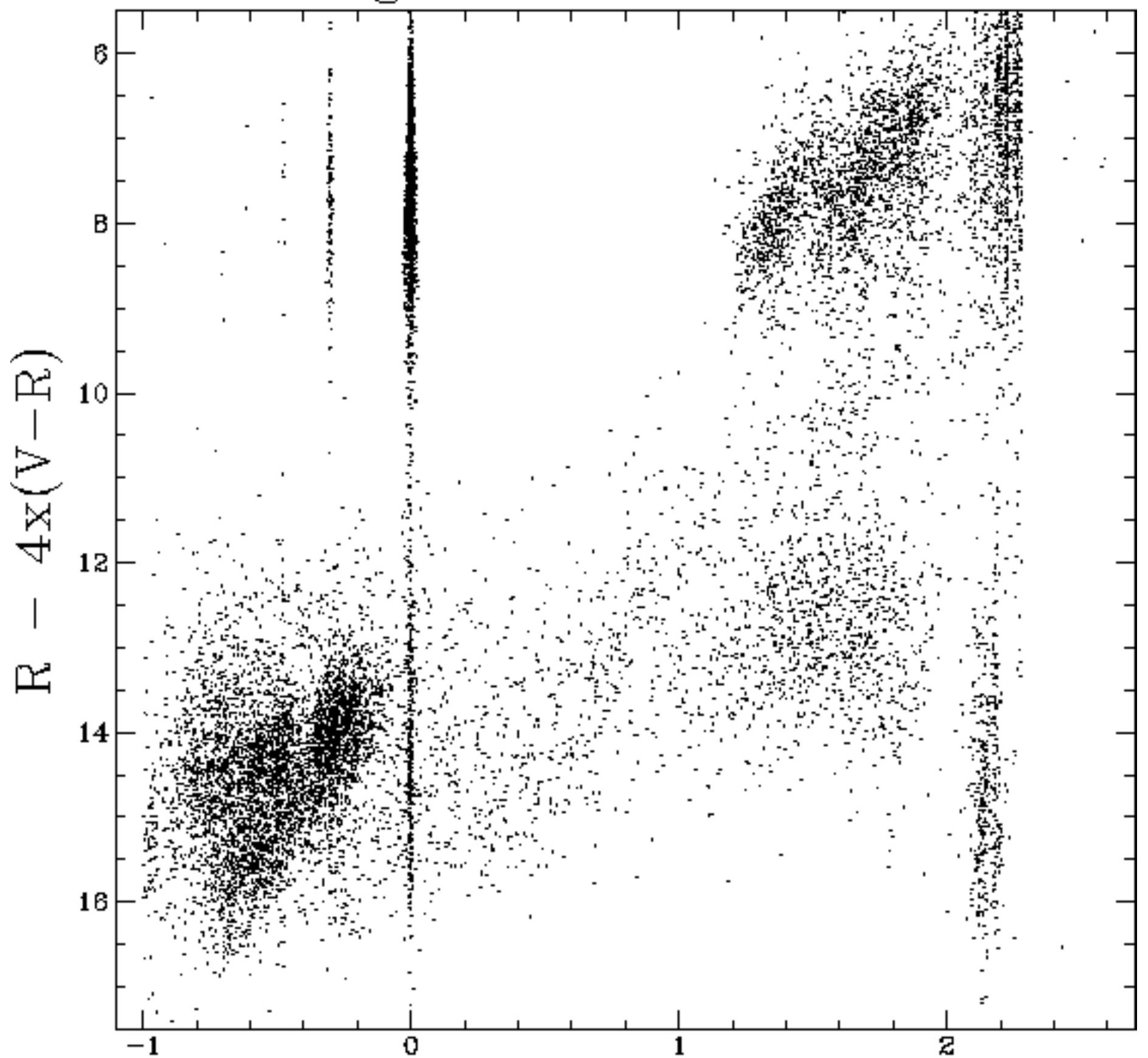




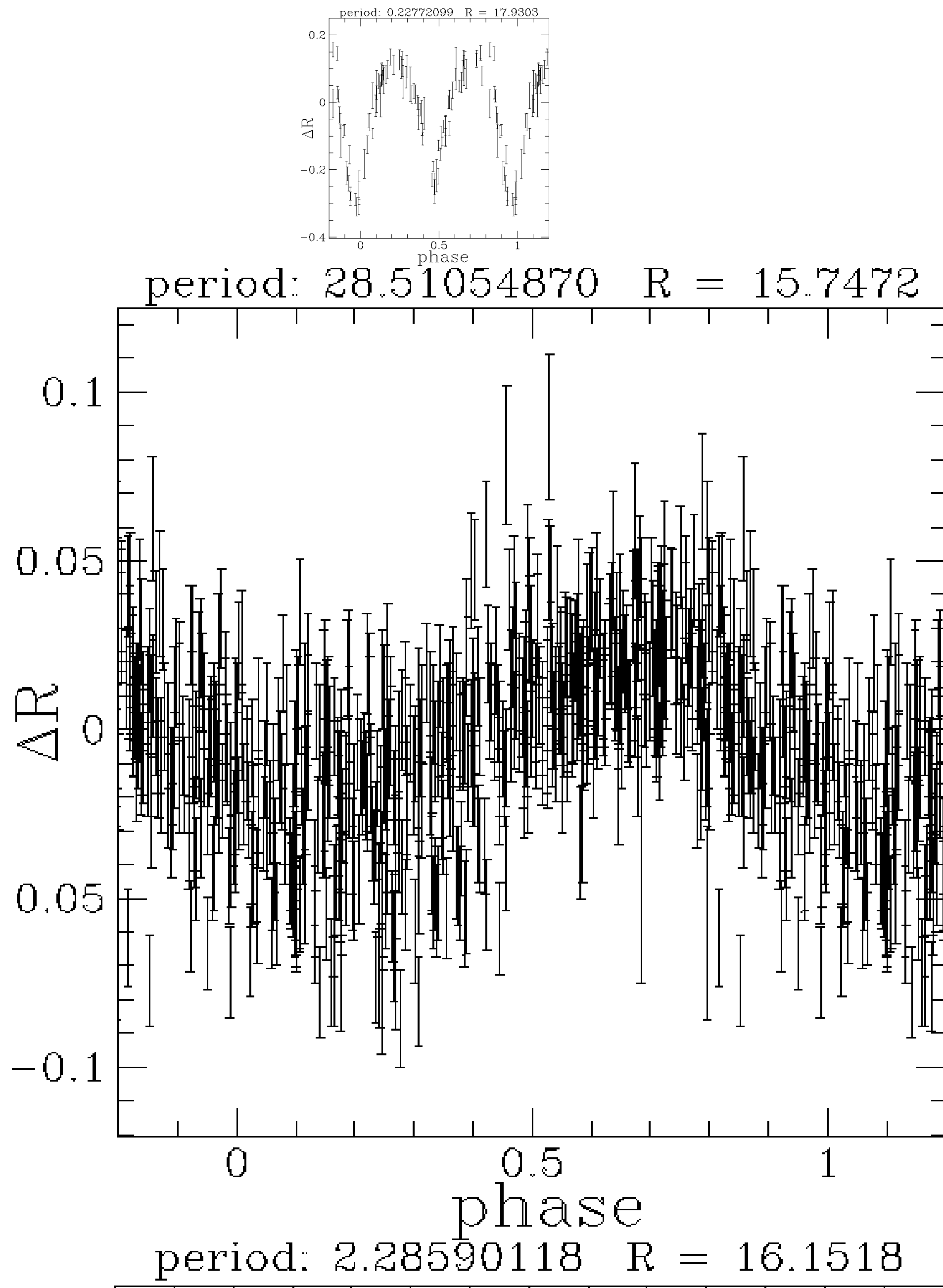




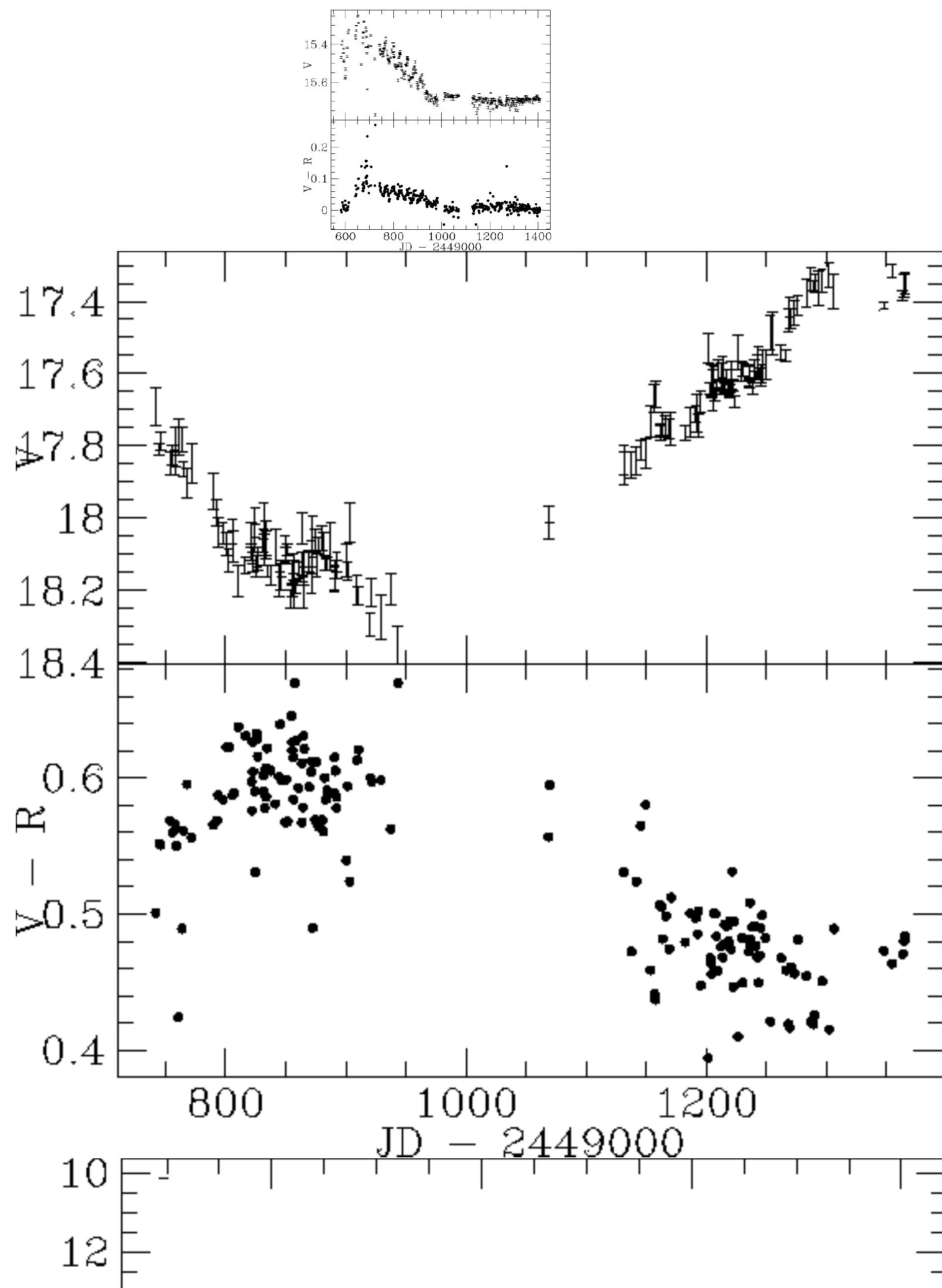

